\title{
Correlation of the venous angioarchitecture of multiple cerebral cavernous malformations with familial or sporadic disease: a susceptibility-weighted imaging study with 7-Tesla MRI
}

\author{
Philipp Dammann, MD, ${ }^{1}$ Karsten Wrede, MD, ${ }^{1}$ Yuan Zhu, PhD, ${ }^{1}$ Toshinori Matsushige, MD, ${ }^{1,2}$ \\ Stefan Maderwald, PhD, ${ }^{3}$ Lale Umutlu, MD, ${ }^{3,4}$ Harald H. Quick, PhD, ${ }^{3,5}$ Ute Hehr, MD, ${ }^{6}$ \\ Matthias Rath, MD, ${ }^{7}$ Mark E. Ladd, PhD, ${ }^{3,8}$ Ute Felbor, MD, ${ }^{7}$ and Ulrich Sure, MD ${ }^{1}$ \\ ${ }^{1}$ Department of Neurosurgery, ${ }^{4}$ nstitute for Diagnostic and Interventional Radiology and Neuroradiology, and ${ }^{5}$ High Field \\ and Hybrid MR Imaging, University Hospital Essen; ${ }^{3}$ Erwin L. Hahn Institute for Magnetic Resonance Imaging, University of \\ Duisburg-Essen; ${ }^{6}$ Center for and Department of Human Genetics, University of Regensburg; ${ }^{7}$ Department of Human Genetics, \\ University Medicine Greifswald and Interfaculty Institute of Genetics and Functional Genomics, University of Greifswald; \\ ${ }^{8}$ Division of Medical Physics in Radiology, German Cancer Research Center (DKFZ), Heidelberg, Germany; and 2Department of \\ Neurosurgery, Graduate School of Biomedical and Health Sciences, Hiroshima University, Hiroshima, Japan
}

OBJECTIVE Multiple cerebral cavernous malformations (CCMs) are rare lesions that occur in sporadic or familial form. Depending on the disease form, the natural history and treatment of the lesions strongly vary. Molecular analysis of an underlying germline mutation (CCM1-3) is the most sensitive screening method to distinguish between sporadic and familial cases. However, based on the different pathomechanisms that are believed to be involved in either form, significant distinctions in the CCM-associated cerebral venous angioarchitecture should be detectable. This has not been systematically studied.

METHODS A consecutive series of 28 patients with multiple CCMs (681 total) diagnosed on 1.5-T MRI underwent genetic screening for CCM1-3 mutations and high-resolution susceptibility-weighted imaging (SWI) of the cerebral venous angioarchitecture with 7-T MRI. Imaging data were analyzed to examine the CCM-associated venous angioarchitecture. Results were correlated with findings of molecular analysis for CCM1-3 mutations.

RESULTS Two different SWI patterns (sporadic and familial) were found. The presence of associated developmental venous anomalies correlated with negative screening for germline mutations ( 11 sporadic) in all cases. All patients with confirmed familial disease showed normal underlying venous angioarchitecture. Additionally, a very unusual case of a probable somatic mutation is presented.

CONCLUSIONS The SWI results of the venous angioarchitecture of multiple CCMs correlate with sporadic or familial disease. These results are consistent with the theory that venous anomalies are causative for the sporadic form of multiple CCMs.

http://thejns.org/doi/abs/10.3171/2016.2.JNS152322

KEY WORDS CCM; susceptibility-weighted imaging; SWl; high-field MRI; magnetic resonance imaging; cerebral cavernous malformations; familial disease; familial cavernomatosis; CCM1-3; vascular disorders

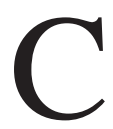
EREBRAL cavernous malformations (CCMs) are low-flow focal vascular abnormalities, which are histologically characterized as closely packed thin-walled vessels filled with blood at different stages of thrombosis. Recurrent intra- or extralesional (micro) hemorrhages and secondary iron deposits determine the clinical findings in patients with symptoms, mainly seizures or focal neurological deficits. ${ }^{15}$ Cerebral cavernous malformations affect approximately $0.5 \%$ of the population and occur mostly as a sporadic and less frequently

ABBREVIATIONS CCM = cerebral cavernous malformation; DVA = developmental venous anomaly; FA = flip angle; MLPA = multiplex ligation-dependent probe amplification; $R$ = in-plane resolution; $S T$ = slice thickness; $S W I$ = susceptibility-weighted imaging.

SUBMITTED October 6, 2015. ACCEPTED February 11, 2016.

INCLUDE WHEN CITING Published online May 6, 2016; DOI: 10.3171/2016.2.JNS152322. 
as an inherited autosomal-dominant (familial) form. ${ }^{27}$ Whereas the former is usually characterized by single lesions, the latter presents with multiple lesions distributed throughout the entire CNS and/or other organ systems. ${ }^{30}$ However, depending on the specific underlying germline mutation within CCM1/KRIT1, CCM2/MGC4607, or $C C M 3 / P D C D 10,{ }^{26}$ penetrance of the disease, age of the patient, and other factors (e.g., applied MRI technique), the number of detectable lesions strongly varies, ranging from a single or a few lesions to hundreds of CCMs ${ }^{14} \mathrm{On}$ the other hand, sporadic CCMs may present as multiple lesions as well $(10 \%-15 \%)$, with an underlying germline mutation emerging in up to two-thirds of the cases. ${ }^{26,32}$

Because a familial form has a significant impact on the natural history and treatment of the disease and especially a considerable social component compared with the sporadic form, early clarification during consultation of a patient with multiple lesions $(\geq 2)$ is desirable. Molecular screening for known $C C M 1-3$ mutations is the most sensitive method but is often initiated only months to years later., ${ }^{7,26,32}$ Additionally, information on a potential family history may be fragmentary, especially because many CCMs remain asymptomatic. ${ }^{15}$ Consequently, the initial MRI diagnosis of multiple CCMs frequently leads to a prolonged situation of uncertainty for the patient and their family.

However, based on the distinct pathomechanisms suspected to be involved in the formation of sporadic and familial CCMs (venous malformation or developmental venous anomaly $[D V A]^{29}$-associated formation ${ }^{2}$ or lossof-function mutation [germline or somatic] in 1 of the 3 $C C M$ genes with consecutive endothelial dysfunction, ${ }^{33}$ respectively), specific differences in local venous angioarchitecture ${ }^{6,21,25}$ between these forms of multiple CCMs are expected to be detectable. This has not been systematically studied. We propose the following hypotheses: that 1) a differing distribution of multiple CCMs and 2) a differing adjacent venous angioarchitecture of multiple CCMs can be detected, and 3) that these will correlate with genetic screening for an underlying familial disease.

Therefore, we conducted a study to search for specific patterns in high-resolution MRI of multiple CCMs $(\geq 2)$. To increase the sensitivity for delineation of CCM lesions and associated venous vascular structures, ${ }^{5}$ all MRI was performed at 7-T magnetic field strength ${ }^{13}$ using susceptibility-weighted imaging (SWI $)^{10,20}$ and standard anatomical imaging protocols. After image analysis, the results were matched with findings from genetic examination that included screening for $C C M 1-3$ gene mutations.

\section{Methods}

The study was conducted at the Department of Neurosurgery and the Erwin L. Hahn Institute for Magnetic Resonance Imaging of the University of Duisburg-Essen, Germany, in accordance with all guidelines set forth by the approving institutional review board. The study was performed as part of a superordinate fundamental research study on high-field MRI. Informed consent according to the Declaration of Helsinki was obtained from every patient before examination. Sequence analysis of $C C M 1-3$ genes was mainly performed at the Department of Human Genetics, University Medicine Greifswald, Germany, and in part at the Center for and Department of Human Genetics, University of Regensburg, Germany.

\section{Patient Characteristics}

All patients who were admitted to our department between 2010 and 2015 who showed multiple CCMs $(\geq 2)$ on 1.5-T MRI (contrast-enhanced T1-weighted, T1-weighted, T2-weighted, and $\mathrm{T} 2 *$-weighted) and who had undergone gene sequence analysis of CCMI-3 were included in this study and underwent SWI with 7-T MRI. Patients with incomplete genetic screening, pregnant patients, and patients with a history of cranial radiation were excluded. Patients with the sporadic form of the disease and solitary lesions were analyzed in a previous study. ${ }^{6}$

\section{Methods Used}

Basic clinical data and medical history of all patients were assessed according to the reporting standards of CCM research. ${ }^{1}$

\section{The 7-Tesla MRI Protocol}

In all patients, SWI was performed at $7 \mathrm{~T}$ (Magnetom 7T; Siemens Healthcare). Susceptibility-weighted imaging sequences were established in previous work. ${ }^{5}$ The whole-body ultra-high-field MR system was equipped with a single-channel transmitter/32-channel receiver head coil (Nova Medical), and a gradient system capable of $45 \mathrm{mT} / \mathrm{m}$ maximum amplitude and a slew rate of 220 $\mathrm{mT} / \mathrm{m} / \mathrm{msec}$. The SWI parameters were as follows: TE 15 msec, TR $27 \mathrm{msec}$, flip angle (FA) $14^{\circ}$, in-plane resolution (R) $250 \times 250 \mu^{2}$, slice thickness (ST) $1.5 \mathrm{~mm}$, and bandwidth $140 \mathrm{~Hz} /$ pixel. The SWI data were processed to phase, magnitude, susceptibility, and minimum intensity projection images. In addition, anatomical T1-weighted (TR $2500 \mathrm{msec}$, TE $1.4 \mathrm{msec}, \mathrm{FA} 6^{\circ}, 0.7 \mathrm{~mm}$ isotropic), T2-weighted (TR $6000 \mathrm{msec}$, TE $99 \mathrm{msec}, \mathrm{FA} \mathrm{29}{ }^{\circ}$, ST $3 \mathrm{~mm}, \mathrm{R} 0.5 \mathrm{~mm}^{2}$ ), and time-of-flight angiography (TR $20 \mathrm{msec}$, TE $4.3 \mathrm{msec}$, FA $20^{\circ}$, ST $0.4 \mathrm{~mm}, \mathrm{R} 0.2 \mathrm{~mm}^{2}$ ) sequences were acquired.

\section{Image Analysis}

All 7-T MR images (phase, magnitude, SWI, T1-weighted, and T2-weighted) were analyzed using an in-house workstation by 2 observers experienced in SWI analysis. The following criteria were assessed: number, location, type, ${ }^{28}$ and size of the CCMs ( $\geq 1 \mathrm{CCM}$ exceeding or not exceeding $10 \mathrm{~mm}$ ). Size was determined using axial plane T2-weighted turbo spin echo. Also, distribution over 1, 2, or $\geq 3$ regions of the brain (frontal, temporal, occipital, parietal lobe, insular lobe, basal ganglia, brainstem, and cerebellum) was determined. A distribution over $>2$ regions of the brain was defined as a multifocal distribution.

In brain regions with $\mathrm{CCMs}$, the local venous angioarchitecture was inspected for absence or presence of a typical DVA or anatomical abnormalities as specified previously, ${ }^{6}$ i.e., presence of transcerebral medullary veins or formation of venous radicles. In cases of a confirmed DVA, the number of collecting veins and presence of ste- 
TABLE 1. Schematic representation of cerebral venous system sections

\begin{tabular}{cl}
\hline \multicolumn{1}{c}{ Venous System } & \multicolumn{1}{c}{ Sections } \\
\hline Superficial veins & Superior \\
& Superficial sylvian \\
& Deep sylvian \\
& Inferior \\
& Inferior anastomotic \\
& Superior anastomotic \\
\hline Deep veins & Great \\
& Basal \\
& Internal \\
\hline Subependymal veins & Fissura transversa \\
& Lateral ventricle \\
& Cisterna tecti \\
\hline Infratentorial veins & Medial pontine \\
& Lateral pontine \\
& Superficial cerebellar \\
& Deep cerebellar \\
\hline
\end{tabular}

nosis (vessel diameter reduction $>50 \%)^{12}$ or an angulated course $\left(<90^{\circ} \text { angulation }\right)^{12}$ of collecting veins were assessed. Developmental venous anomalies were grouped as small, medium, or large based on involvement of 2, up to 4 , or $>4$ venous system sections, respectively. The classification of the venous system used as a physiological reference is shown in Table $1 .{ }^{31}$ Susceptibility-weighted and time-of-flight images were correlated to distinguish between arterial and venous vessels, e.g., for cases of arterialized venous malformations.

In a subgroup of patients for whom SW images at 1.5 $\mathrm{T}$ were available $(\mathrm{n}=25)$, the following criteria were assessed and compared with findings at 7-T MRI: 1) number of CCMs, and 2) absence or presence of a DVA.

\section{Molecular Genetic Analyses}

Sanger sequencing analyses of individuals with CCMs were performed with informed consent as described in Spiegler et al..$^{32}$ Mutation-negative individuals were subsequently screened for large $C C M 1-3$ alterations using multiplex ligation-dependent probe amplification (MLPA; SAL-
SA MLPA Kits P130 and P131, MRC Holland). ${ }^{8}$ GenBank and ENSEMBL accession numbers were as follows: $C C M 1$ (GenBank:NM_194456.1, Ensembl:ENST00000394507), CCM2 (GenBank:NM_031443.3, Ensembl:ENST0000025 8781), and CCM3 (GenBank:NM_145860.1, Ensembl: ENST00000392750). Sequences were analyzed in SeqPilot with the ENSEMBL data sets. DNA mutation numbering was based on the cDNA sequence, with +1 corresponding to the A of the ATG translation initiation codon.

\section{Statistical Analysis}

Statistical analysis was performed using SPSS Statistics version 22 (IBM Corp.). Interval-scaled data were expressed as the mean and SD, and nominal data were expressed as absolute numbers and valid percent. Data were tested for normal distribution by conducting a Shapiro-Wilk test, in addition to histograms and Q-Q plots. Intergroup comparison was performed with the Fisher exact test for categorical variables and the Mann-Whitney U-test for continuous variables. To estimate the long-run probability of predicting a familial or sporadic disease based on the MRI data of our sample, the rule of 3 was applied (95\% CI). ${ }^{11}$

\section{Results}

Twenty-eight patients ( 15 women and 13 men) with a mean age of $42.7 \pm 12.9$ years (range 19-64 years) were included in the study (Tables 2 and 3). Six additional patients who presented with multiple CCMs between 2010 and 2015 did not meet the eligibility criteria. Genetic sequence analysis was performed in all 28 patients. Two patients were brothers from a family with known $C C M 3 \mathrm{mu}-$ tation (Cases 4 and 8; Table 3), and another 2 patients were mother and daughter from a family with known CCM2 mutation (Cases 2 and 13). Twenty-four patients presented with at least 1 symptomatic hemorrhage, including 4 patients suffering from cavernoma-related epilepsy. Four patients were asymptomatic.

\section{Image Analysis}

At 7-T SWI, the overall number of CCMs was 681. The mean number of CCMs was $22.4 \pm 40.0$ (range 2-153). A

TABLE 2. Baseline clinical data, MRI findings, and genetic screening of patients with cluster-type MRI pattern

\begin{tabular}{rccccccc}
\hline Case No. & Age (yrs) & Sex & No. of CCMs & Multifocal Distribution & Associated DVA & Family History & Genetic Screening \\
\hline 1 & 38 & F & 5 & Yes $^{*}$ & Yes & No & Negative \\
\hline 2 & 57 & M & 4 & No & Yes & No & Negative \\
\hline 3 & 55 & F & 8 & No & Yes & No & Negative \\
\hline 4 & 52 & F & 4 & No & Yes & No & Negative \\
\hline 5 & 45 & F & 5 & No & Yes & No & Negative \\
\hline 6 & 44 & F & 3 & No & Yes & No & Negative \\
\hline 7 & 36 & M & 3 & No & Yes & No & Negative \\
\hline 8 & 57 & M & 6 & No & Yes & No & Negative \\
\hline 9 & 61 & M & 5 & No & Yes & No & Negative \\
\hline 10 & 41 & F & 3 & No & Yes & No & Negative \\
\hline 11 & 34 & M & 6 & Yes & No & Negative \\
\hline
\end{tabular}

* Patient showed multifocal DVA with associated CCM involving both hemispheres and the posterior fossa. 
TABLE 3. Baseline clinical data, MRI findings, and genetic screening of patients with scattered-type MRI pattern

\begin{tabular}{|c|c|c|c|c|c|c|c|c|}
\hline $\begin{array}{l}\text { Case } \\
\text { No. }\end{array}$ & $\begin{array}{l}\text { Age } \\
\text { (yrs) }\end{array}$ & Sex & $\begin{array}{l}\text { No. of } \\
\text { CCMs }\end{array}$ & $\begin{array}{l}\text { Multifocal } \\
\text { Distribution }\end{array}$ & $\begin{array}{c}\text { Associated } \\
\text { DVA }\end{array}$ & $\begin{array}{l}\text { Family } \\
\text { History }\end{array}$ & $\begin{array}{c}\text { Genetic } \\
\text { Screening }\end{array}$ & CCM1-3 Mutation \\
\hline 1 & 51 & M & 153 & Yes & No & Yes & Positive & CCM1:C.1258G>T \\
\hline 2 & 21 & $\mathrm{~F}$ & 2 & Not & No & No & Positive & CCM2:C.30G>A \\
\hline 3 & 54 & $\mathrm{~F}$ & 85 & Yes & No & No & Positive & CCM1:c.1730+4_1730+7delAGTA \\
\hline 4 & 23 & M & 23 & Yes & No & Yes & Positive & CCM3:c.334_337delCAAA \\
\hline 5 & 45 & $\mathrm{~F}$ & 21 & Yes & No & Yes & Positive & CCM2:C.55C>T \\
\hline 6 & 45 & M & 14 & Yes & No & Yes & Positive & CCM1:C.1890G>A \\
\hline 7 & 19 & $\mathrm{~F}$ & 4 & Not & No & Yes & Positive & CCM1:c.730-1G>A \\
\hline 8 & 28 & M & 19 & Yes & No & Yes & Positive & CCM3:c.334_337delCAAA \\
\hline 9 & 33 & $\mathrm{~F}$ & 85 & Yesł & No & No & Negative & - \\
\hline 10 & 25 & M & 11 & Yes & No & No & Positive & CCM2:205-1G>A \\
\hline 11 & 47 & M & 4 & Yes & No & No & Positive & CCM3:c.63_64dupCC \\
\hline 12 & 64 & M & 132 & Yes & No & No & Positive & CCM1:C.1391G>A \\
\hline 13 & 53 & $\mathrm{~F}$ & 6 & Yes & No & No & Positive & CCM2:C.30G>A \\
\hline 14 & 56 & M & 3 & Yes & No & Yes & Positive & CCM1:C.12_20del \\
\hline 15 & 24 & $\mathrm{~F}$ & 4 & Yes & No & Yes & Positive & CCM1:_deletion_of_entire_gene \\
\hline 16 & 43 & $\mathrm{~F}$ & 3 & Yes & No & Yes & Positive & CCM1:C.1780_1783delins \\
\hline 17 & 47 & $\mathrm{~F}$ & 6 & Yes & No & No & Negative & - \\
\hline
\end{tabular}

multifocal distribution was found in 17 cases. Association of the CCMs with a typical DVA was found in 11 cases. Further CCMs associated with local venous abnormalities were not detected. In 16 cases, at least $1 \mathrm{CCM}$ exceeded $10 \mathrm{~mm}$. According to the Zabramski classification, ${ }^{34}$ Type Ia/b was found in 19 patients (33 CCMs), Type II in 14 patients (30 CCMs), Type III in 2 patients (4 CCMs), and Type IV in 1 patient. Six hundred thirteen lesions were visible as a small signal cancellation on SWI only. Intralesional or extralesional macro hemorrhage $(>10 \mathrm{~mm})$ was found in 9 cases.

Developmental venous anomalies showed $2.5 \pm 1.9$ (range 1-7) collecting veins. In 9 DVAs a stenosis was confirmed, and in 9 DVAs an angulated course of the collecting vein was confirmed. Eight were categorized as large, and 3 as medium. Further details are shown in Table 4.

According to MRI criteria, the patients were subdivided into 2 groups: (multi)focal distribution without associated DVA (scattered type; Fig. 1) and focal distribution with associated DVA (cluster type; Fig. 2). For details, see Tables 2 and 3. A schematic of the patterns is provided in Fig. 3.

At 1.5-T SWI $(\mathrm{n}=25)$, the overall number of detected CCMs was lower compared with 7-T MRI (mean $33.2 \pm$ 41.3 vs $39.8 \pm 49.2$ lesions). Fewer lesions were detected in 6 patients. The detection rate of DVA was similar between the 2 imaging modalities.

\section{Molecular Genetic Analysis}

Sanger sequencing and MLPA revealed a CCMI-3 mutation in 15 patients, of whom 6 showed no previous family history. However, 1 of these 6 inherited the mutation from her mother, who was discovered to have 6 asympto- matic CCMs at the age of 52 years (Cases 2 and 13; Table $3)$. In 13 patients without previous family history, Sanger sequencing and MLPA did not reveal a mutation. For details see Tables 2 and 3 .

\section{Type of MRI Pattern}

The cluster-type MRI pattern was not associated with a positive molecular screening for $C C M 1-3$ in any case. The scattered-type MRI pattern showed a positive genetic screening in 15 of 17 cases. This difference was statistically significant (Fisher's exact test, $\mathrm{p}<0.0001$ ).

The overall number of CCMs was lower in the cluster-

TABLE 4. Specifications of DVAs

\begin{tabular}{ccccccc}
\hline $\begin{array}{c}\text { Case } \\
\text { No. }\end{array}$ & $\begin{array}{c}\text { Location } \\
\text { (IT vs } \\
\text { ST) }\end{array}$ & $\begin{array}{c}\text { No. of } \\
\text { CVs }\end{array}$ & Stenosis & $\begin{array}{c}\text { Angulated } \\
\text { Course }\end{array}$ & $\begin{array}{c}\text { Drainage } \\
\text { (deep vs } \\
\text { sup) }\end{array}$ & Size \\
\hline 1 & Both & 7 & Yes & Yes & Deep & Large \\
\hline 2 & ST & 3 & No & Yes & Sup & Large \\
\hline 3 & IT & 3 & Yes & Yes & Both & Large \\
\hline 4 & Both & 2 & No & Yes & Deep & Large \\
\hline 5 & IT & 1 & Yes & Yes & Sup & Large \\
\hline 6 & IT & 1 & Yes & No & Sup & Large \\
\hline 7 & ST & 1 & Yes & Yes & Sup & Medium \\
\hline 8 & ST & 1 & Yes & Yes & Deep & Medium \\
\hline 9 & IT & 3 & Yes & Yes & Sup & Large \\
\hline 10 & ST & 1 & Yes & No & Sup & Medium \\
\hline 11 & Both & 4 & Yes & Yes & Both & Large \\
\hline
\end{tabular}

CV = collecting vein; IT = infratentorial; ST = supratentorial; sup= superficial. 

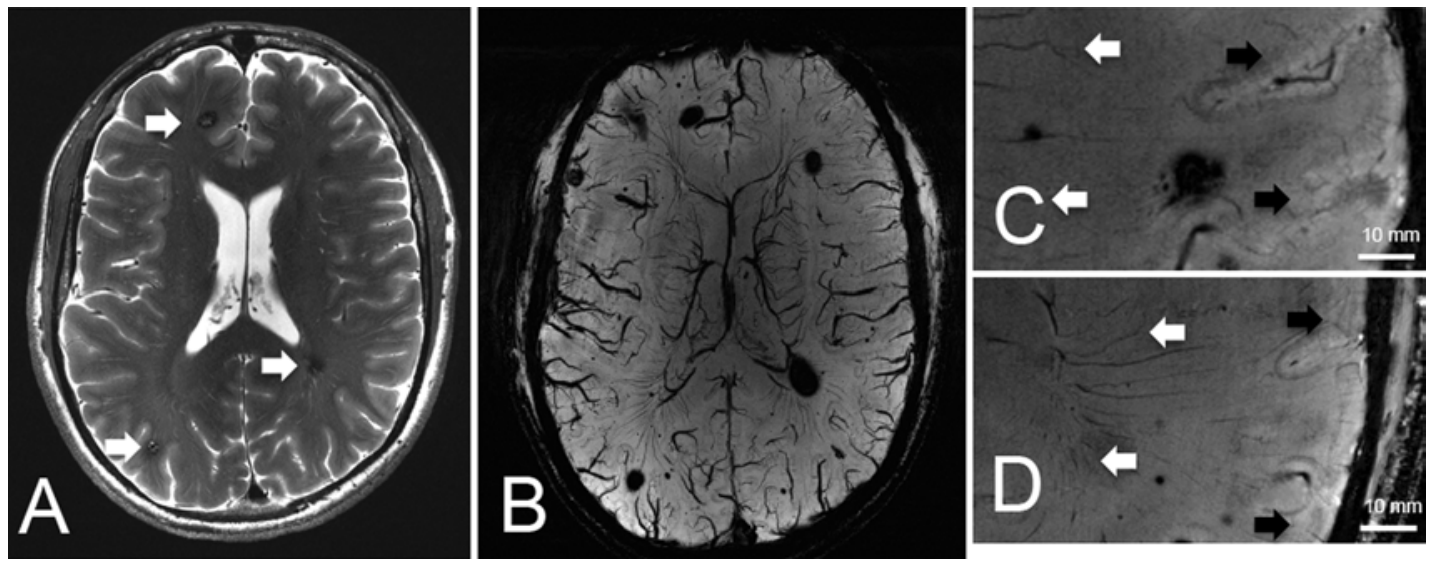

FIG. 1. A scattered-type MRI pattern is shown. A: A 7-T T2-weighted image depicting multiple supratentorial CCMs in a typical popcorn-like appearance with hemosiderin rim (arrows). B: A 7-T SW image (minimum intensity projection) revealing additional small CCMs but normal underlying venous anatomy. This represents a typical example of a random-type pattern of multiple CCMs. C and D: Normal venous cortical (black arrows) and subcortical (white arrows) ultrastructures are shown. The CCMs appear as bubble-like lesions without larger outflow vessels.

type group compared with the random-type group $(4.7 \pm$ 1.5 vs $33.8 \pm 48.5$; Mann-Whitney U-test, $\mathrm{p}=0.07)$. Other categorical variables (hemorrhage size $>10 \mathrm{~mm}$ and CCM type) showed no significant difference. Based on the rule of 3 , the long-run probability to fail in the prediction of sporadic versus familial disease based on the MRI data of our sample is, from a mere statistical perspective, approximately $10.7 \%(95 \% \mathrm{CI}){ }^{11}$

\section{Discussion}

In this study, the MRI pattern of multiple CCMs
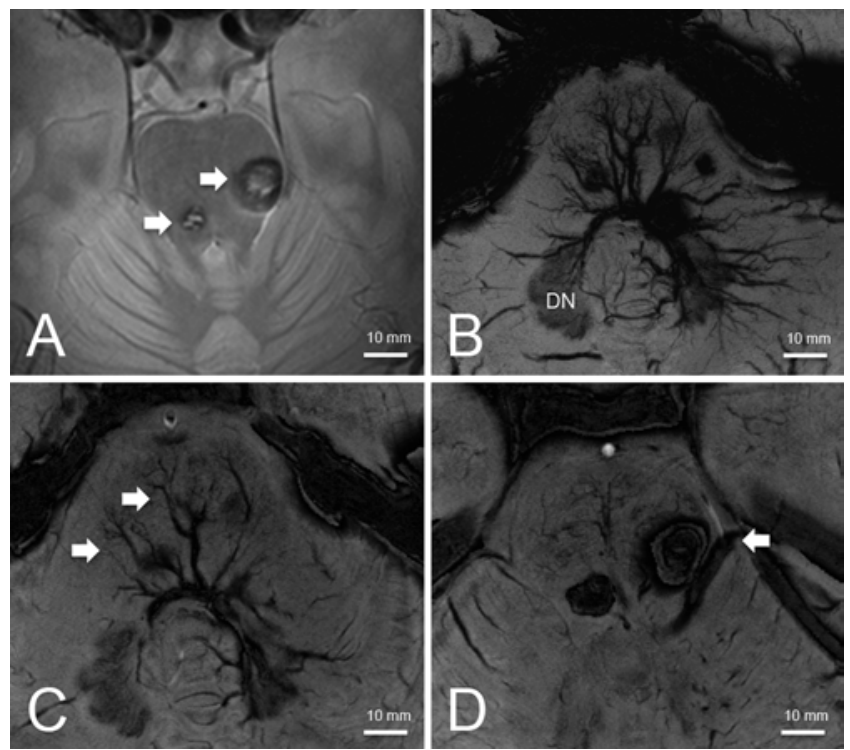

FIG. 2. A cluster-type MRI pattern is shown. A: 7-T T2-weighted images depicting multiple CCMs of the brainstem in a typical popcorn-like appearance with hemosiderin rim (white arrows). B-D: A 7-T SW images (minimum intensity projection) revealing the large underlying DVA with caput medusae formation. This represents a typical example of a cluster-type pattern of multiple CCMs. Note the completely disarranged venous architecture with enlarged venous radicles (C, white arrows) and angulated and stenosed ( $\mathrm{D}$, white arrow) collecting vein. $\mathrm{DN}=$ dentate nucleus. clearly differed between suspected sporadic (negative genetic screening) and confirmed familial (positive genetic screening) forms of the disease. Disease distribution in suspected sporadic CCMs was limited to 1 or 2 regions of the brain, except in those patients with multifocal DVAs (Cases 1 and 11; Table 2). In confirmed familial disease, CCMs were distributed over multiple regions of the brain, except for 2 cases in which only a few lesions ( 2 and 4 CCMs, respectively) were present (Cases 2 and 7; Table 3 ). Accordingly, overall, fewer lesions were found in sporadic cases compared with familial cases. However, half of patients with confirmed familial disease showed a lesion burden of $<10$ CCMs.

Most importantly, the presence of a typical DVA with the CCMs located in the draining territory of the collecting vein was associated with a negative genetic screening in all cases, indicating a sporadic disease. An associated typical DVA or local venous abnormality was not found in any of the confirmed familial cases. These differences were statistically significant. There was no difference in the number of lesions showing acute hemorrhage (Types Ia and Ib vs Types II, III, and IV) or in the size of the lesions between the 2 groups. Based on the repetitive patterns of distribution over the brain and association/nonassociation with DVA, we suggest naming the patterns cluster-type (sporadic form) and scattered-type (familial form).

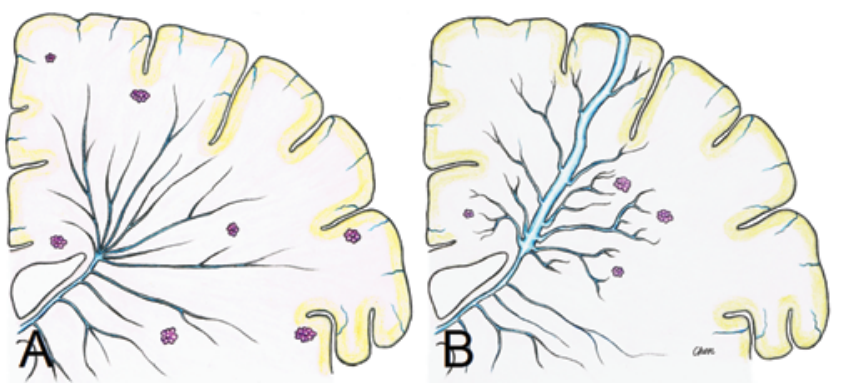

FIG. 3. A schematic of scattered-type (A) and cluster-type (B) MRI patterns of multiple CCMs. Copyright Bixia Chen. Published with permission. 


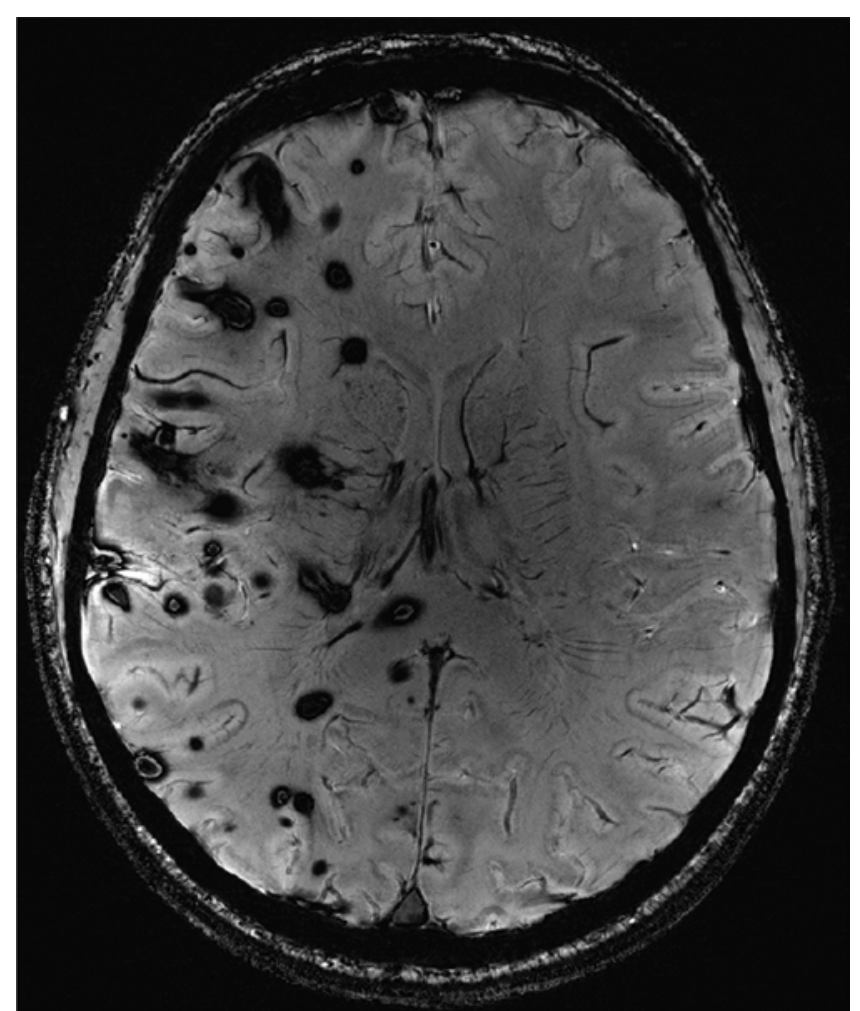

FIG. 4. Case illustration. A 7-T SW image (minimum intensity projection) of multiple CCMs, localized to 1 hemisphere with normal underlying venous anatomy.

Our results indicate that in patients with cluster-type distribution, the existence of an underlying familial form of the disease is extremely unlikely. On the other hand, a scattered-type distribution is suggestive of familial disease. Still, in 2 cases of scattered-type MRI, routine genetic screening was negative for a CCM1-3 mutation along with a negative family history. Interestingly, one of these patients (Case 9; Table 2) shows an extraordinary pattern of multiple CCMs exclusively localized to 1 hemisphere (Fig. 4). Such a pattern of distribution would be rather suggestive of an underlying somatic mutation. A somatic mutation occurs during development and propagates in subsequent tissues. Recently, McDonald et al. reported evidence for somatic mosaicism in a similar case..$^{18}$

Nevertheless, for patients with scattered-type MRI, definite diagnosis of a germline mutation requires genetic analysis. Such analysis nowadays identifies increasing numbers of already published mutations but may also very rarely be hampered by diagnostic gaps. These gaps may be explained by the existence of undetected mosaicism, an additional fourth $C C M$ gene, ${ }^{16}$ or deep intronic mutations that could activate alternative or cryptic splice sites. ${ }^{23}$ These technical limitations are generally thought to be responsible for an underlying mutation remaining undiscovered..$^{21,26,32}$ We attribute the negative genetic screening of Case 17 (Table 3) to such diagnostic gaps.

In our department, we still generally recommend genetic screening in patients with multiple CCMs, especially in younger patients or in families with symptomatic rela- tives. On the other hand, we already inform patients about the lower probability of a familial disease if a cluster-type MRI pattern is shown.

The association of DVA and multiple CCMs in sporadic and familial cases has been reported before. The series of Mondéjar et al., ${ }^{21}$ in which pathogenic mutations were screened for in familial and sporadic cases in Spain and Portugal, included 4 cases that presented multiple CCMs with associated DVA. None of these carried a pathogenic mutation in the $C C M$ genes. The MRI study of Petersen et al. ${ }^{25}$ reported a nonassociation of DVA in familial cases as well. However, the series included families with CCMI/ KRIT1 mutation only (genetic screening was not available in all patients), compiled both solitary and multiple lesions, and did not provide SWI or further analysis of MRI patterns.

Susceptibility-weighted imaging, especially when performed with high-field-strength MRI, is more sensitive for CCM lesions and DVA compared with clinical routine imaging (T2* weighted) and can be applied at submillimeter resolution. ${ }^{5}$ This minimizes the possibility of missing any vascular lesions. However, in the present study, relevant MRI data (detection of DVA and detection of CCM lesions) did not show a significant difference between 1.5$\mathrm{T}$ and 7-T MRI, indicating that this information will be available from routine clinical protocols in most cases (at least for large or medium DVAs, as found in this study; very subtle DVAs may be missed at lower field strength). A clear advantage of SWI for the detection of DVAs is that no exogenous contrast agent is necessary.

Finally, our findings (cluster-type pattern) support previous assumptions that DVAs may be associated with the formation of sporadic CCMs, as judged by their systematic co-occurrence and close topographical relationship. There exist various reports of de novo CCM formation adjacent to the draining territory of a DVA..$^{3,4,17}$ Furthermore, our own group has found systematic association of CCM and DVA in a consecutive series of sporadic, mainly solitary, lesions. ${ }^{6}$ These findings argue for a divergent pathogenesis of sporadic and familial CCMs. ${ }^{24,33}$ Awad et al. discussed this in further detail in their hemorrhagic angiogenic proliferation hypothesis. ${ }^{2}$ According to the hypothesis, structural and hemodynamic conditions within the DVA are causative factors. In fact, in this series, we could confirm the presence of specific angioarchitectural factors of the DVAs (stenosis and angulated course of draining veins) that are believed to be involved in the formation of concurrent CCMs. ${ }^{12}$

In contrast, familial CCMs are derived from systematic endothelial dysfunction due to a loss-of-function mutation within CCMI-3; the most common form (CCM1/KRITI) involves the RhoA and Rho kinase system. ${ }^{9}$ Pharmacological therapy affecting this system, a suggested future therapeutic option for familial cases, ${ }^{19}$ may thus not be suitable for the much more common sporadic cases.

Our study examines high-resolution SWI combined with genetic screening in a prospective consecutive series of patients with multiple CCMs for the first time. The main study limitation is a relatively small sample size. However, multiple CCMs are rare, accounting for only $15 \%$ of all $\mathrm{CCMs}$, which generally leads to small series. 


\section{Conclusions}

Our results indicate a systematic association of multiple CCMs with DVAs in the sporadic form of the disease. If such a cluster-type pattern is diagnosed on MRI, familial disease, with its severe clinical implications, thus seems rather unlikely. This is of high relevance in the counseling of patients. Especially when combined with routine genetic screening, the presence of these specific MRI patterns may therefore further ensure the diagnosis of a familial or sporadic disease in patients with multiple CCMs.

Finally, our results are consistent with the theory that DVAs are causative for the formation of (1 form of) sporadic CCMs, which has a significant impact on the natural history and treatment of the disease. Of course, because these findings are preliminary and based on a small sample size, they must be verified in future studies.

\section{Acknowledgments}

We thank Dr. Bixia Chen, Department of Neurosurgery, University Hospital Essen, for the illustration of Figure 3. Drs. Dammann and Wrede were funded by a Clinician Scientist Fellowship of the Medical Faculty of the University of Duisburg-Essen, Germany (IFORES Grant).

\section{References}

1. Al-Shahi Salman R, Berg MJ, Morrison L, Awad IA: Hemorrhage from cavernous malformations of the brain: definition and reporting standards. Stroke 39:3222-3230, 2008

2. Awad IA, Robinson JR Jr, Mohanty S, Estes ML: Mixed vascular malformations of the brain: clinical and pathogenetic considerations. Neurosurgery 33:179-188, 1993

3. Cakirer S: De novo formation of a cavernous malformation of the brain in the presence of a developmental venous anomaly. Clin Radiol 58:251-256, 2003

4. Campeau NG, Lane JI: De novo development of a lesion with the appearance of a cavernous malformation adjacent to an existing developmental venous anomaly. AJNR Am J Neuroradiol 26:156-159, 2005

5. Dammann P, Barth M, Zhu Y, Maderwald S, Schlamann M, Ladd ME, et al: Susceptibility weighted magnetic resonance imaging of cerebral cavernous malformations: prospects, drawbacks, and first experience at ultra-high field strength (7-Tesla) magnetic resonance imaging. Neurosurg Focus 29(3):E5, 2010

6. Dammann P, Wrede KH, Maderwald S, El Hindy N, Mueller $\mathrm{O}$, Chen B, et al: The venous angioarchitecture of sporadic cerebral cavernous malformations: a susceptibility weighted imaging study at 7 T MRI. J Neurol Neurosurg Psychiatry 84:194-200, 2013

7. Faurobert E, Albiges-Rizo C: Recent insights into cerebral cavernous malformations: a complex jigsaw puzzle under construction. FEBS J 277:1084-1096, 2010

8. Gaetzner S, Stahl S, Sürücü O, Schaafhausen A, HalligerKeller B, Bertalanffy H, et al: CCM1 gene deletion identified by MLPA in cerebral cavernous malformation. Neurosurg Rev 30:155-160, 2007

9. Glading A, Han J, Stockton RA, Ginsberg MH: KRIT-1/ CCM1 is a Rap1 effector that regulates endothelial cell cell junctions. J Cell Biol 179:247-254, 2007

10. Haacke EM, Mittal S, Wu Z, Neelavalli J, Cheng YC: Susceptibility-weighted imaging: technical aspects and clinical applications, part 1. AJNR Am J Neuroradiol 30:19-30, 2009

11. Hanley JA, Lippman-Hand A: If nothing goes wrong, is everything all right? Interpreting zero numerators. JAMA 249:1743-1745, 1983
12. Hong YJ, Chung TS, Suh SH, Park CH, Tomar G, Seo KD, et al: The angioarchitectural factors of the cerebral developmental venous anomaly; can they be the causes of concurrent sporadic cavernous malformation? Neuroradiology 52:883891,2010

13. Kraff O, Fischer A, Nagel AM, Mönninghoff C, Ladd ME: MRI at 7 Tesla and above: demonstrated and potential capabilities. J Magn Reson Imaging 41:13-33, 2015

14. Laurans MS, DiLuna ML, Shin D, Niazi F, Voorhees JR, Nelson-Williams C, et al: Mutational analysis of 206 families with cavernous malformations. J Neurosurg 99:38-43, 2003

15. Lee KS, Spetzler RF: Cerebral cavernous malformations. Arch Neurol 46:1273, 1989

16. Liquori CL, Berg MJ, Squitieri F, Ottenbacher M, Sorlie M, Leedom TP, et al: Low frequency of PDCD10 mutations in a panel of CCM3 probands: potential for a fourth CCM locus. Hum Mutat 27:118, 2006

17. Maeder P, Gudinchet F, Meuli R, de Tribolet N: Development of a cavernous malformation of the brain. AJNR Am J Neuroradiol 19:1141-1143, 1998

18. McDonald DA, Shi C, Shenkar R, Gallione CJ, Akers AL, Li $\mathrm{S}$, et al: Lesions from patients with sporadic cerebral cavernous malformations harbor somatic mutations in the CCM genes: evidence for a common biochemical pathway for CCM pathogenesis. Hum Mol Genet 23:4357-4370, 2014

19. McDonald DA, Shi C, Shenkar R, Stockton RA, Liu F, Ginsberg MH, et al: Fasudil decreases lesion burden in a murine model of cerebral cavernous malformation disease. Stroke 43:571-574, 2012

20. Mittal S, Wu Z, Neelavalli J, Haacke EM: Susceptibilityweighted imaging: technical aspects and clinical applications, part 2. AJNR Am J Neuroradiol 30:232-252, 2009

21. Mondéjar R, Solano F, Rubio R, Delgado M, Pérez-Sempere A, González-Meneses A, et al: Mutation prevalence of cerebral cavernous malformation genes in Spanish patients. PLoS One 9:e86286, 2014

22. Otten P, Pizzolato GP, Rilliet B, Berney J: [131 cases of cavernous angioma (cavernomas) of the CNS, discovered by retrospective analysis of 24,535 autopsies.] Neurochirurgie 35:82-83, 128-131, 1989 (Fr)

23. Pagani F, Baralle FE: Genomic variants in exons and introns: identifying the splicing spoilers. Nat Rev Genet 5:389-396, 2004

24. Pagenstecher A, Stahl S, Sure U, Felbor U: A two-hit mechanism causes cerebral cavernous malformations: complete inactivation of CCM1, CCM2 or CCM3 in affected endothelial cells. Hum Mol Genet 18:911-918, 2009

25. Petersen TA, Morrison LA, Schrader RM, Hart BL: Familial versus sporadic cavernous malformations: differences in developmental venous anomaly association and lesion phenotype. AJNR Am J Neuroradiol 31:377-382, 2010

26. Riant F, Cecillon M, Saugier-Veber P, Tournier-Lasserve E: CCM molecular screening in a diagnosis context: novel unclassified variants leading to abnormal splicing and importance of large deletions. Neurogenetics 14:133-141, 2013

27. Rigamonti D, Hadley MN, Drayer BP, Johnson PC, HoenigRigamonti K, Knight JT, et al: Cerebral cavernous malformations. Incidence and familial occurrence. N Engl J Med 319:343-347, 1988

28. Rigamonti D, Spetzler RF, Drayer BP, Bojanowski WM, Hodak J, Rigamonti KH, et al: Appearance of venous malformations on magnetic resonance imaging. J Neurosurg 69:535-539, 1988

29. Ruíz DS, Yilmaz H, Gailloud P: Cerebral developmental venous anomalies: current concepts. Ann Neurol 66:271-283, 2009

30. Rutka JT, Brant-Zawadzki M, Wilson CB, Rosenblum ML: Familial cavernous malformations. Diagnostic potential of magnetic resonance imaging. Surg Neurol 29:467-474, 1988 
31. Seeger W: Microsurgery of Cerebral Veins. Vienna: Springer, 1984

32. Spiegler S, Najm J, Liu J, Gkalympoudis S, Schröder W, Borck $\mathrm{G}$, et al: High mutation detection rates in cerebral cavernous malformation upon stringent inclusion criteria: one-third of probands are minors. Mol Genet Genomic Med 2:176-185, 2014

33. Yadla S, Jabbour PM, Shenkar R, Shi C, Campbell PG, Awad IA: Cerebral cavernous malformations as a disease of vascular permeability: from bench to bedside with caution. Neurosurg Focus 29(3):E4, 2010

34. Zabramski JM, Wascher TM, Spetzler RF, Johnson B, Golfinos J, Drayer BP, et al: The natural history of familial cavernous malformations: results of an ongoing study. J Neurosurg 80:422-432, 1994

\section{Disclosures}

The authors report no conflict of interest concerning the materi- als or methods used in this study or the findings specified in this paper.

\section{Author Contributions}

Conception and design: Dammann Acquisition of data: Dammann, Wrede, Matsushige, Maderwald, Umutlu, Quick, Hehr, Rath, Ladd, Felbor. Analysis and interpretation of data: Dammann, Wrede, Zhu, Umutlu, Rath, Felbor. Drafting the article: Dammann, Wrede, Quick, Felbor, Sure. Critically revising the article: Zhu, Matsushige, Quick, Hehr, Ladd, Sure. Approved the final version of the manuscript on behalf of all authors: Dammann. Study supervision: Sure.

\section{Correspondence}

Philipp Dammann, Department of Neurosurgery, University Hospital Essen, Hufelandstrasse 55, 45122 Essen, Germany. email: philipp.dammann@uk-essen.de. 